the too partial examination of the subject before us, which has been conducted by various observers.

Mr. Nasmyth promises to prosecute an extended series of researches into every branch of odontology; in doing so we feel sure that he will reconcile many of the present conflicting opinions, and we hope to receive from his hands, what is much wanted in medical literature, a Systematic Treatise on the Development, Structure, and Diseases of the Teeth.

To conclude: to those who wish to pursue the subject which we have so briefly brought under notice, we recommend the first part of the work of Mr. Nasmyth, as containing an entire translation of the papers of Retzius, which are illustrated by many beautiful and original plates; also a complete view of the researches of those whose names we have introduced in the present article; and, lastly, a comprehensive historical survey of all works on odontology.-Brit. and Foreign Med. Review.

\title{
REMARKS ON THE PERCEPTION OF FORM.
}

(Concluded from page 125.)

Fory results from the limitation of the extension of bodies, and it may be proved by admeasurement that the particular form of any body is determined by the relative distance and direction of the points of its surface from its centre, or from any other of its points, always measuring from the same point. In regular forms, as a perfect sphere, it is easy to prove this. The form of a crystal depends on the distance and direction of the particles of its surface from the primitive nucleus of formation.

It is my present object to show that the apparent form of a body depends on the relative distance and direction of the points of its visible surface from the point of observation, when we see it correctly, and that when we are deceived with regard either to the relative distance or the relative direction of its points, its form appears different from what it really is. If this can be shown, it will necessarily follow that the perception of form is compound, and that it cannot, in accordance with the received principles of phrenology, be attributed to a single faculty; for it is one of its fundamental principles that every perception essentially distinct in its nature, depends on a distinct power of the mind.

In investigations like the present, it must be constantly borne in mind that the impulses by which bodies at a distance make impressions on our organs of sight, come from them in the form of rays; thus when we look at an object, rays of light of different lengths, and coming from different directions, strike the retina, and the inpressions they there make being propagated to the brain, excite the perception of the distance and direction of the points from which they come. When we touch any object, the impressions it makes on us are conveyed by a very circuitous route along the nerves to the brain, where their character is perceived by the different mental faculties; but our perceptions are the same as if they came to the brain in right lines, and it is only by observation and

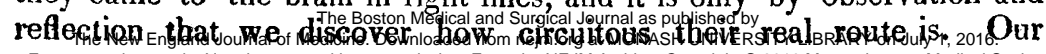


zerceptive faculties are so constituted that they take no direct cognizance $3 i^{-}$the refraction of the rays of light, or of sound, or of that of the imzulses of touch; our perceptions are what they would be if the impulses zame in a direct line from the points from which they appear to come.

I draw a line four inches in length, which appears straight, and which san be proved mathematically to be straight; I place a point which I call the point of observation three inches from it, and equidistant from soth of its extremities, and I find that if I vary perceptibly the distance and direction of any of its middle points from the point of observation, Thile the others remain the same, the line ceases to appear straight, and that in reality it ceases to be straight. If I wish to vary the position of is extreme points, or to add new points to its extremities, and still preserve the straightness of the line, I find that I am obliged to place them in a certain direction and at a certain distance from the point of observation. A straight line may be proved in the following manner. $\because$ A straight line is that line the distance of each of whose points from the point of observation is proportionate to the secant of the direction of this point. Understanding by the direction of this point the angle $\mathrm{P} A \mathrm{~B}$, which the line A $\mathbf{P}$ drawn to it makes with the perpendicular A B." Draw a line from the point of observation to the middle of the straight line, and another to one of its extremities. Place $A$ at the point of observation, $\mathbf{B}$ at the middle of the straight line, and $\mathbf{P}$ at the extremity to which the other line is drawn.

When I look at a plane surface two inches square, placing its central point immediately opposite, and each of its angles equally distant from, the centre of the cornea, I perceive that its points become more and more distant from the centre of the cornea, in proportion to their distance from the centre of the square, and that though there are complete circles of points, all equally distant from the centre of the cornea, each point is in a different line of direction. If I vary perceptibly the distance of a single point, while the others remain the same, the surface ceases to appear plane and to be plane. If I substitute for the plane surface a sphere two inches in diameter, I perceive that the distance of its points from the centre of the cornea increases much more rapidly as they recede from the centre of the spherical surface than they did in the plane surface. When I substitute for the sphere a regular cone whose base is two inches in diameter, $\mathbf{I}$ perceive that the lines of direction of the points of its surface increase in length from the point of observation much more rapidly as they recede from the vertex, than they would if the surface were spherical. If I vary perceptibly the distance or direction of a single point, while the others remain the same, the form appears altered, and that it is really altered may be proved by admeasurement. It is evident, then, that when a person knows the relative distance and direction of all the points of the visible surface of a body from any given point, he has a perfect knowledge of its visible form. It is undeniable that we do get our knowledge of the visible properties of bodies by means of impulses coming in the form of rays from every point of their visible surface, and it is also undeniable that the apparent form of a body depends on the apparent relative distance 
and direction of the points of its visible surface from the point of observation; for it has been, and can again be, proved by innumerable experiments, that when we are deceived with regard either to the relative distance or relative direction of the points of a surface, its form appears different from what it is. When we can succeed in getting two distinct images of an object which appear to come from entirely different distances and directions, the object appears double. This may be done by pushing a little aside one of the eyes when they are both directed towards the same object; the images are then formed on non-corresponding parts of the retina, and in consequence appear to come from entirely different distances and directions, but the form of the images is the same, because the relative distance and direction of their parts are the same in both. When we place a straight rod in a vessel, and cover a part of it with water, it appears bent, and the reason is that the relative distance and direction of the points of its surface appear changed on account of the refraction of the rays of light coming from the part under the water, and we can never by any effort learn to see the rod straight, while it and the eye remain in the position referred to.

When we look between two long parallel rows of trees or buildings, they appear gradually to approach each other at their further extremities. This deception results from the limited nature of our faculties, and from the laws of light. We cannot estimate by sight a great distance so accurately as we can a short one; other things being the same, we receive fewer rays of light from a distant object than we do from a near one, and in consequence of the diminution of the vividness of the impressions, we cannot perceive so accurately their character. The points of the lines appear nearer than they are, but they still appear in their true lines of direction, when the rays are not refracted before they reach the eye. The deception with regard to their distance increases so gradually and so regularly, that the apparent lines have all the characters of a right line, and of course do not appear bent; in other words, the lines appear to have exactly that inclination towards the eye which they would require to have to constitute them right lines, were they situated as they appear to be. We are deceived as to the real distance of the points of each line, but not as to their relative distance, from the point of observation.

When we are deceived with regard to the form of a body by shading, we are always deceived with regard to the relative distance or direction of its points, from the point of observation, commonly both. The reader may satisfy himself of this by examining prints or paintings. The labor of the artist being laid out on a flat surface, he is obliged to throw depth into breadth or height, and he who succeeds in representing the most naturally depth by breadth or height, is the best artist, as far as concerns perspective.

As (other things being the same) a body always appears smaller the further it is removed from the eye, it seems that size and distance are essentially the same perception-if so, they must depend on the same faculty. Length is distance from one end to the other; breadth, width,

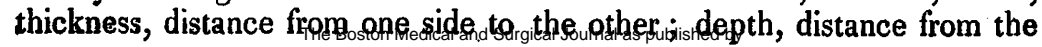


op to the bottom; height, distance from the bottom to the top; these - ords therefore include the idea of direction. Distance is perceived by we faculty called size, and direction by that called locality.

The reader will perceive by the following extract from the last edition Mr. Combe's "System of Phrenology," that my opinion with regard - the perception of form is not altogether without phrenological support. = In the last edition, I mentioned the case of a lady who having Form brge and Size deficient, copied figures accurately in regard to form, but maccurately in regard to size. To which statement Mr. Jeffrey objected wat size is necessary to proportion, and proportion to form, and that mere was inconsistency in the account of the lady's talents. Mr. Jeffrey is right : she informs me that it is only the simplest forms, which have iew parts, that she is able to copy correctly, and in drawing even them she will err in size; but that when a figure has detached parts, although sine may give the outline of each part by itself with considerable sccuracy, it will be larger or smaller than the original; whence the whole figure will be deficient in proportion. In drawing from nature, she failed in perspective; nevertheless she feels great pleasure in observing forms, recollects them easily, has a complete mental consciousness of the powers of Form and Size being different, and of the one being strong and the other weak in her mind." It seems to me that this case furnishes very strong evidence in favor of the opinion that the perception of form does not depend on a single faculty. It was to be expected that the lady would err less in drawing a small part of a complex figure than in drawing the whole of it, and it is evident that she did not recollect forms accurately.

When phrenologists assert, that other things being the same, the person whose eyes are the furthest apart has the most accurate and distinct perception, and consequently the best recollection of forms, they assert no more than can be proved to be correct; but when they go on to say that the perception of form depends on a single cerebral organ, which, when large, produces breadth between the eyes, I think they assert more than can be proved. There are certain physical reasous why a person whose eyes are far apart perceives the form of objects more accurately and with less trouble than a person whose eyes are near together, supposing every other thing to be the same. It is an easily demonstrable fact that those persons who bave the eyes far apart, are able to get a more extensive, and consequently a more accurate, riew of many irregular bodies, at a single glance, and without moving the head, than those persons whose eyes are near together. It is easy to place a body in such a manner that a man whose eyes are far apart shall see three faces of it, while another, whose eyes are near together, shall see only one face, when both are standing in the same position. It is evident, then, that the two men would have very different ideas of its form. Persons whose eyes are near together may see distinctly what is in view, but their range of vision is less extensive, and their ideas of the form of many irregular bodies cannot be so accurate, as they rould be were their eyes far apart; on the same principle that a man who is near sighted and can see only the lower part of a building, has a very 
different idea of it than he would have if he could see it all. From the constitution of the material world, a man who has but one eye sees much less than he would if he had two. The portraits of a painter whose eyes are near together are apt to appear flat and dead; they want relief and depth; he sees less of the face and head at a single view when in certain positions, than he would if his eyes were far apart, and he is naturally inclined to represent as much as he sees at once, or but little more. Were he aware of the disadvantage under which he labors, he would find it difficult to overcome it by superior attention, and at the same time make his portraits appear natural. He would be apt to destroy their unity. Gilbert Stuart's portraits are remarkable for their depth and relief; they appear to stand out from the canvass; you imagine you can see the entire form of the person represented. Though I do not know it to have been the case, I have no doubt that Stuart's eyes were uncommonly far apart.

The organ of Size, when large, evidently increases the distance between the eyes, particularly when Individuality is also large and sinks low between them. When Individuality is small, Size may be large without pushing the eyes apart, for in such cases it is generally placed higher than when Individuality is large. There may be considerable breadth between the eyes when Individuality is large and Size deficient. Let the phrenologist look at the portraits of Sterne, Captain Cook, Reubens, Lord Bacon, given in Dr. Spurzheim's work on phrenology, and he will be convinced that if the portraits are correct, the organ of Size has much influence on the position of the eye-balls. From an examination of skulls externally and internally, and of the living head, I am convinced that the distance at which the eyes are placed from one another depends very much on the position and development of the organs of Size and Individuality.

$$
\text { Boston, Sept., } 1839 . \quad \text { Andrew Alexander, M.D. }
$$

\section{SMALLPOX IN MAINE.}

BY A CORRESPONDENT.

Dear Sir,-I have recently visited, by request of their physicians and municipal authorities, the towns of Boothbay and Edgecomb, to examine several cases of snallpox, which were found to be of the distinct variety. It was introduced into Boothbay by a seaman landed there, who came directly from some infected region, and he passed through the different stages of the disease without exciting even a suspicion of its character; it having passed among the inhabitants for chickenpox. The case was a very mild one-pustules distinct and few in number, and the fever slight. In the immediate neighborhood of this case there have been several of patients who have recovered (one with the loss of an eye), and there were yesterday, under the care of Dr. Cushman (a quondam pupil of mine) 20 cases in which the eruption had appeared, and others coming on; and I regret to add that the malignity of the disease increases with the number of cases. Some of these cases were very severe. High fever during the suppurative stage-great tumefaction of the face

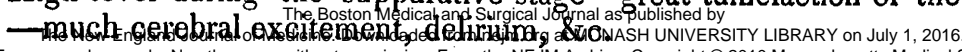

